\title{
Radio Detection of Cosmic Rays at the Auger Engineering Radio Array
}

\author{
Klaus WEIDENHAUPT ${ }^{* 1}$ for the Pierre Auger Collaboration ${ }^{2}$ \\ ${ }^{1}$ RWTH Aachen University \\ E-mail: weidenhaupt@physik.rwth-aachen.de \\ 2 Observatorio Pierre Auger, Av. San Martín Norte 304, 5613 Malargüe, Argentina \\ Full author list: http://www.auger.org/archive/authors_2014_04.html
}

\begin{abstract}
The Pierre Auger Observatory detects ultra-high energy cosmic rays by measuring extensive air showers induced in the earth's atmosphere. Besides established detection techniques, using a $3000 \mathrm{~km}^{2}$ array of particle detectors sampling shower particles at ground level, and detecting fluorescence light emitted during the shower development with telescopes the Observatory explores the potential of radio detection of cosmic rays with the Auger Engineering Radio Array (AERA). Radio detection has the potential to provide information on cosmic ray shower properties with a duty-cycle not limited by day and moon light as in the case of the fluorescence technique.

AERA consists of 124 autonomous detector stations sensitive to $\mathrm{MHz}$ frequencies. The stations feature dual-polarized radio antennas, custom low-noise analog and digital electronics and a broad-band wireless communication system. With AERA we face the challenge of self-triggering on the radio pulse in a background dominated environment by implementing various real-time signal processing strategies within the station electronics. We also explore the potential of the radio technique as an integral part of future multi-component detectors by utilizing trigger information from the other Auger detectors and recently, by particle detectors integrated in the radio stations. We will discuss the current cosmic ray measurements and the status and prospects of AERA.
\end{abstract}

Technology and Instrumentation in Particle Physics 2014

2-6 June, 2014

Amsterdam, the Netherlands

\footnotetext{
* Speaker.
} 


\section{Introduction}

Fundamental questions about the nature of cosmic rays remain even 100 years after their discovery. It is still not clear what astrophysical objects accelerate ultra-high energy cosmic rays to extreme energies of more than $10^{20} \mathrm{eV}$ and what is their chemical composition. Furthermore, cosmic rays provide an opportunity to study particle interactions at energies beyond what is accessible by todays man-made accelerators.

The Pierre Auger Observatory addresses these questions by measuring cosmic rays up to the highest energies. As the low flux at these energies requires vast detectors to gather sufficient statistics, the Observatory utilizes the earth's atmosphere as a calorimeter and detects cosmic rays indirectly via their induced air showers. These particle cascades initiated in the upper atmosphere by an inelastic collision of the primary cosmic ray with an air molecule, grow up to billions of particles which partly reach the ground level. The Observatory employs multiple techniques reading out the signature of air showers developing in the "atmospheric calorimeter" to measure cosmic ray properties such as arrival direction, energy and mass.

The fluorescence detectors use imaging cameras to track fluorescence light in the atmosphere that is produced when air molecules are excited by secondary shower particles. The tracked light trace represents a longitudinal profile of the shower development in the atmosphere and allows one to determine the atmospherical depth of the shower maximum which serves as an estimator for the mass of the primary cosmic ray. Fluorescence measurements are restricted to clear moonless nights which reduces their duty cycle to about $14 \%$.

Shower particles that reach ground level are sampled by an array of 1660 water Cherenkov-tanks (cf. Fig. 1) which are distributed over an area of $3000 \mathrm{~km}^{2}$ and form the surface detector of the Observatory.

A promising complementary technique to detect cosmic ray induced air showers via their radio emission is currently being pursuit with the Auger Engineering Radio Array (AERA). About 50 years after first measurements of radio emission from air showers, radio detection has become practical in the last decade for large scale applications, mainly due to technical developments such as fast digital electronics. The detection of the short electric field bursts emitted by air showers are particularly interesting as these signals contain information of the shower development. Radio detection would thus enable a measurement of the shower maximum and therewith the cosmic ray mass without limitations of the duty cycle as for fluorescence detectors.

\section{AERA}

AERA is embedded within the surface detector array of the Pierre Auger Observatory (see Fig. 1) and overlooked by the fluorescence telescopes at the "Coihueco" site. The co-location with the low energy enhancements HEAT [1] and AMIGA [2] maximizes the overlap of different detection techniques and creates a unique environment for complementary studies of cosmic rays. Currently AERA operates 124 radio detector stations on an area of about six $\mathrm{km}^{2}$ (AERA124). In its initial stage AERA consisted of 24 

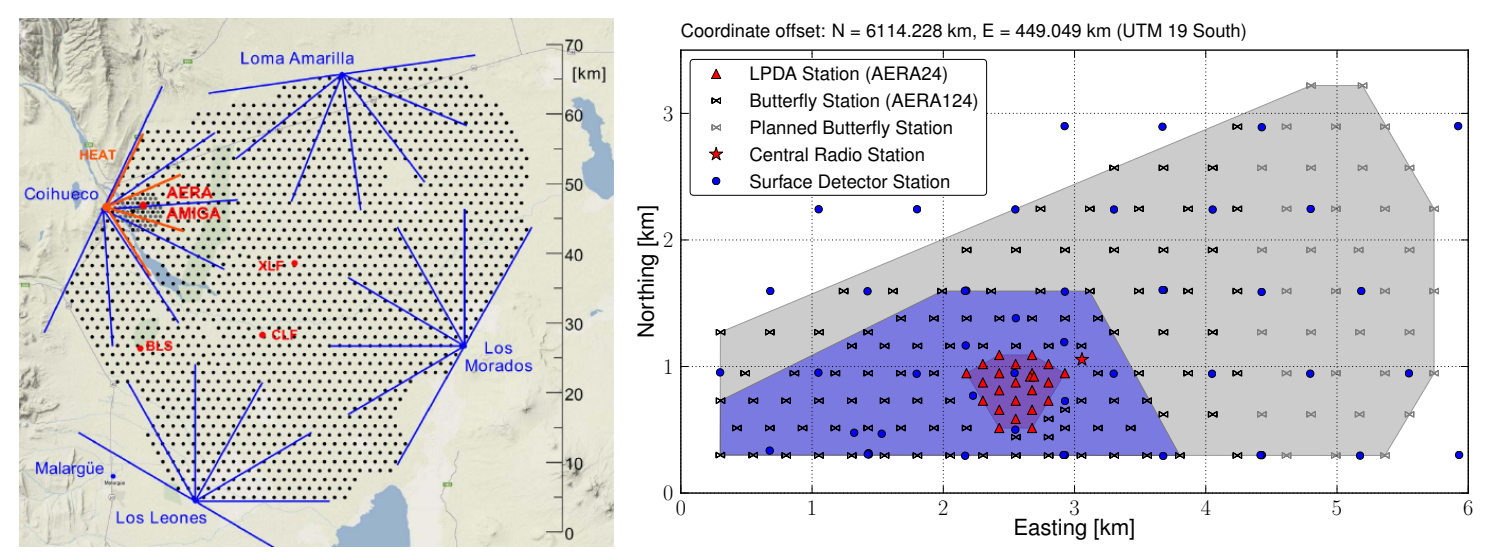

Figure 1: Layout of the Pierre Auger Observatory (left) indicating the positions of the surface detector stations (black dots) and the field of view of the 27 fluorescence telescopes (blue lines). On the right, the layout of AERA is shown. The colored patches highlight the different spacings of the grid of radio detector stations of $150 \mathrm{~m}, 250 \mathrm{~m}$ and $375 \mathrm{~m}$. The Pierre Auger Observatory is located near the town Malarguie in Argentina on an elevated plane about $1400 \mathrm{~m}$ a.s.l..

The layout depicted in Fig. 1 features 2 different types of detector stations. The dense core, named AERA24, corresponds to the first stage of deployment and consists of 24 stations. In a second stage, 100 additional stations following a new design (referred to as AERA124 stations) have been added. The stations are arranged on a hexagonal grid with three different spacings to maximize the number of detected cosmic ray events over a broad energy range. Further AERA124 type stations will be deployed this year. The central radio station (cf. Fig. 1) provides a base for the deployment and hosts parts of the central DAQ of AERA.

The scientific goals of AERA are to understand the details of radio emission processes and to determine the precision of the reconstruction of the cosmic ray properties with the radio technique. Furthermore, hardware and strategies to apply radio detection on large scales are developed, both as a standalone technique and as an integral part of future multi-component detectors.

\section{Radio Detector Stations and Triggering}

The different designs of the AERA24 and AERA124 radio detector stations are shown in Fig. 2. The AERA24 stations follow a more integrated design, optimized towards lower production cost and an easier deployment in large quantities. The sensors to detect the radio emission from air showers are logarithmic periodic dipole antennas for AERA24 and butterfly (bow-tie) antennas for AERA124. Both antenna types cover an ultra-broadband frequency range from $30 \mathrm{MHz}$ to $80 \mathrm{MHz}$ which is essentially free from continuous man-made RF sources such as radio broadcasting. The antennas unify two polarization planes aligned north-south and east-west, respectively, to allow polarization sensitive measurements of the transient electric field. Both antennas operate together with a dedicated low-noise amplifier providing a gain of $18 \mathrm{~dB}$ (AERA24) and $28 \mathrm{~dB}$ (AERA124). A second amplification stage and a bandpass filtering is introduced with a filter amplifier (cf. Fig. 3). 

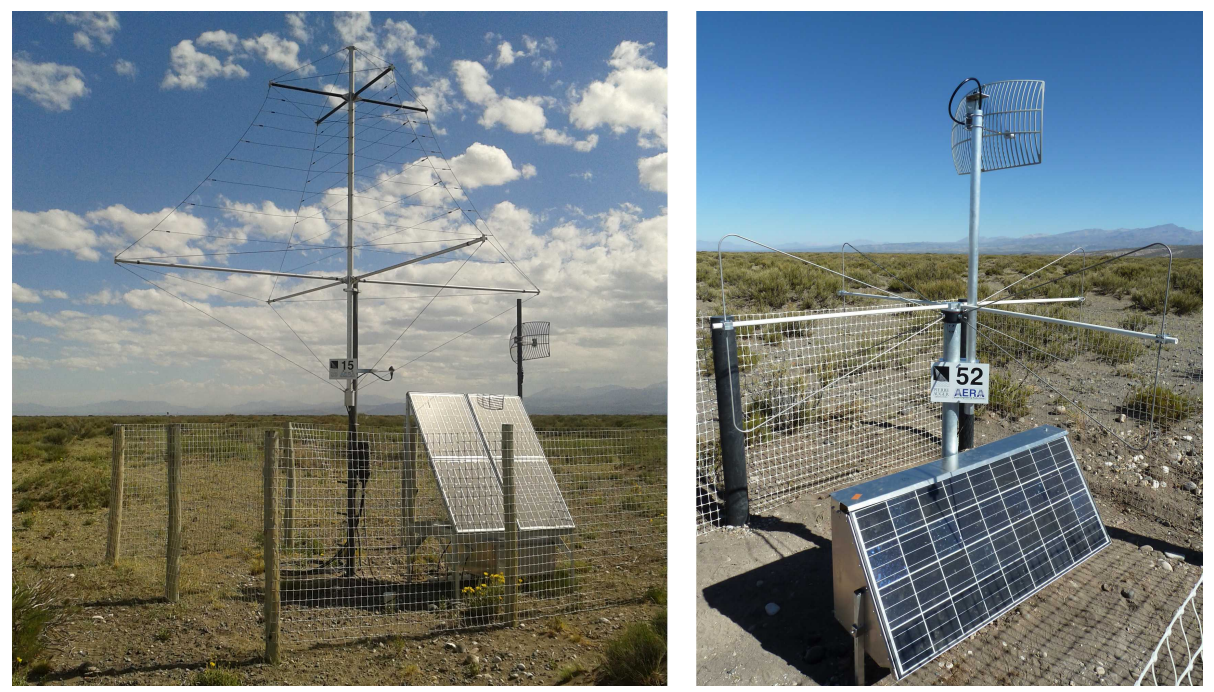

Figure 2: Photograph of the AERA24 (left) and AERA124 (right) radio detector stations. Except the "physics antennas", the dish antennas used for the communication of the AERA stations are visible at the right side of the AERA24 station and at the top of the AERA124 station.

The amplified signals are sampled at $200 \mathrm{MHz}$ with 14 bit ADCs within the custom-made digitizer boards depicted in Fig. 3. The digitizer is equipped with an Altera Cyclone IV FPGA which performs complex data processing such as fast Fourier transformations and allows for a flexible implementation of real-time trigger algorithms. To prevent triggering on noise generated by the station electronics itself, all critical components are shielded by a radio-frequency tight chamber. The communication and data transfer is established with a commercial $5 \mathrm{GHz}$ TDMA (Time Division Multiple Access) wireless link with a bandwidth of $80 \mathrm{Mb} / \mathrm{s}$. An undisturbed signal transfer to the DAQ located in the building of the fluorescence telescope Coihueco at distances of up to $10 \mathrm{~km}$ is guaranteed by high-gain dish antennas visible in Fig. 2. A fully autonomous operation of the stations is enabled by a photovoltaic power supply.

Despite the remote location of AERA, systematic noise studies [3] have revealed various manmade transient noise sources. To face the challenge of self-triggering on the radio pulse in such a background dominated environment, a multi-level trigger approach on both detector station level and on event level, combining the information of multiple detector stations, has been developed. On station level, a multi-threshold time domain trigger [4] filters voltage pulses which show the typical signature of an air shower induced radio pulse. The signal-to-noise ratio of the voltage traces is improved by digital notch filtering of narrow-band continuous signals prior to the triggering. The central DAQ forms an event trigger based on time-coincident station triggers and performs a fast geometric reconstruction of the arrival direction of the pulse. Radio events coming with high rates from distinct directions are then identified as noise pulses and are rejected. Radio events are finally confirmed as cosmic ray signals by coincidences with the Auger baseline detectors.

Besides the self-triggering, the radio stations can be externally triggered by any of the Auger baseline detectors. Furthermore, forty of the AERA124 stations are equipped with a system of scintil- 

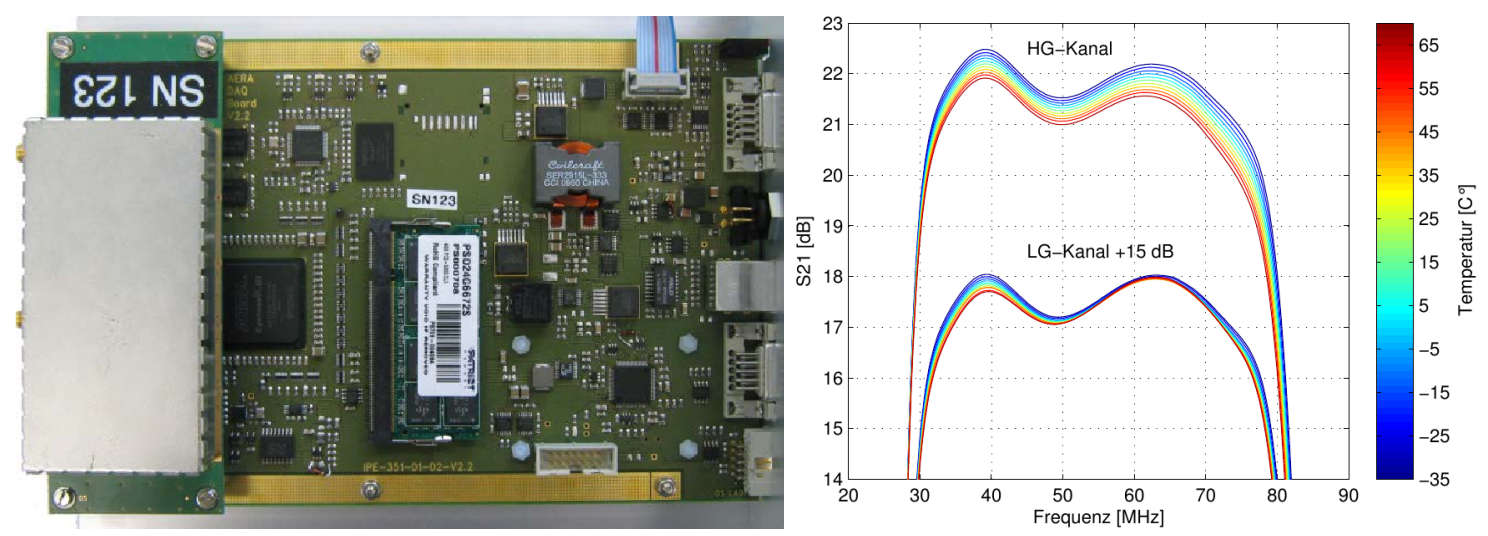

Figure 3: Photograph of the digitizer of the radio detector stations (left) including the filter amplifier mounted as a daughter board. A measurement of the gain of the filter amplifier and its temperature drift is shown on the right. A second type of digitizer which is used in parts of the array and provides similar characteristics is not shown here for the sake of clarity. From [5].

lators providing an internal trigger on secondary shower particles.

\section{Detector Calibration}

In order to perform a reconstruction of the electric field emitted by the air shower based on the measured voltage traces, a calibration of the signal chain and especially of the antenna characteristics is necessary. The filter amplifiers and digitizers are calibrated in a lab by measurements of the scattering parameters (S-Parameters). This allows one to include effects as for instance the temperature variation of the gain of the filter amplifier (which is shown in Fig. 3) in the reconstruction.

The antenna characteristics are fully described in terms of the vector effective length $\vec{H}$ which depends on the incoming direction $(\theta, \phi)$ and the frequency $f$ of the signals

$$
U(\theta, \phi, f)=\vec{H}(\theta, \phi, f) \cdot \vec{E}(\theta, \phi, f) .
$$

This complex quantity gives a direct relation between the impinging electric field vector $\vec{E}$ and the complex voltage $U$ measured at the terminals of the receiving antenna. The commonly known quantity "antenna gain" is related to the magnitude of the vector effective length $|\vec{H}|$. In addition, the phasing of the vector effective length $\phi_{H}=\arg (\vec{H})$ includes a description of the group delay $\tau=-\frac{1}{2 \pi} \frac{d \phi_{H}}{d f}$ imposed by the antenna structure which leads to a dispersion of the measured signals in the time domain.

The vector effective length used for the reconstruction is obtained from simulations with the Nec2 software [6]. Realistic simulation models of the radio detector stations which include not only the response of the antennas itself but also the influence of all conducting station components have been developed and implemented in the Auger reconstruction framework $\overline{\text { Offline [7]. }}$

To verify the simulations and inspect environmental influences such as ground conditions, calibration measurements have been performed at the AERA site. The measurement setup depicted in Fig. 4 is based on transmission measurements between a calibrated signal source and the antenna under test using a vector network analyzer. The transmitter is moved along a predefined path in the 

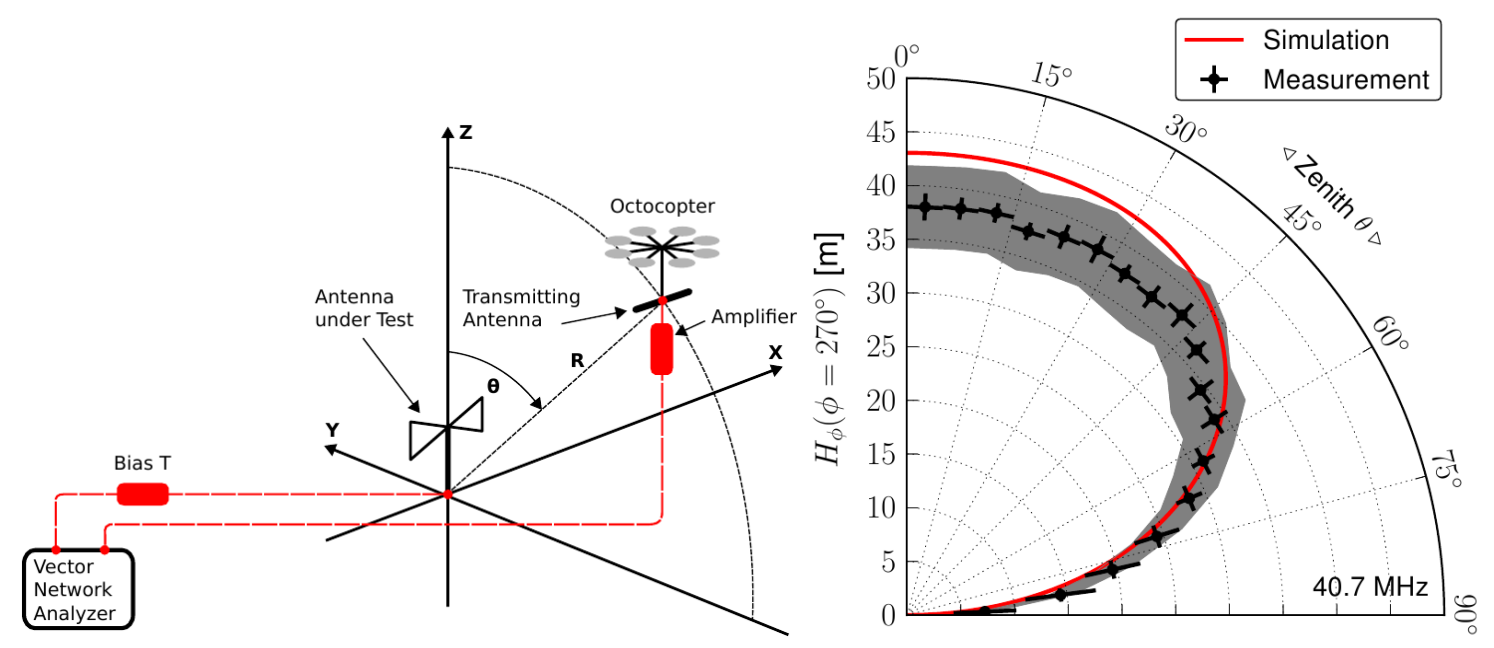

Figure 4: Measurement setup for in situ antenna calibration measurements (left). On the right a measurement of the $e_{\phi}$-component (horizontal polarization) of the magnitude of the vector effective length $\left|\vec{H}_{\phi}\right|$ is shown for the butterfly antenna in comparison to the corresponding simulation. The accessed azimuth angle of $\phi=270^{\circ}$ corresponds to the situation shown in the sketch. The errorbars indicate the statistical uncertainties derived from the spread of data points in zenith bins of $5^{\circ}$. The systematic uncertainties are given by the gray patch.

far-field region, carried by a GPS-controlled flying drone, the octocopter. The zenith dependency of $|\vec{H}|$ is shown in Fig. 4 for the butterfly antenna. A uniform large coverage of the sky and a fair agreement to the corresponding simulations is observed.

\section{Probing Radio Emission Mechanisms}

Among various mechanism contributing to the total emission of radio signals from air showers, the geomagnetic radio emission has been identified as the dominating one by predecessor experiments of AERA such as LOPES [8] and CODALEMA [9]. Charged shower particles are deflected by the earth's magnetic field in opposite directions due to the Lorentz force. Consequently a timevariant transverse dipole evolves within the air shower which is the source of geomagnetic radio emission. The polarization of the emitted electric field vector $\vec{E}$ is then given by the vector product of the shower axis $\vec{n}$ and the magnetic field $\vec{B}$ and the electric field amplitude scales with the angle between the shower axis and the magnetic field $\alpha$

$$
\vec{E} \propto \vec{n} \times \vec{B}, \quad|\vec{E}| \propto|\vec{n}||\vec{B}| \sin (\alpha) .
$$

With the well calibrated detector stations of AERA, the three-dimensional electric field vector can be reconstructed with an unrivaled precision. For cosmic ray events confirmed by coincidences with the Auger surface detector, being measured with AERA24 and having zenith angles smaller than $55^{\circ}$, the measured polarization is shown in the skyplot of Fig. 5 (left). Essentially the same linear polarization is observed in all stations participating in a radio event. Furthermore, only small deviations from the prediction of the geomagnetic emission are found (see Fig. 5 (right)), supporting its dominant role but also suggesting the contribution of second order effects. 

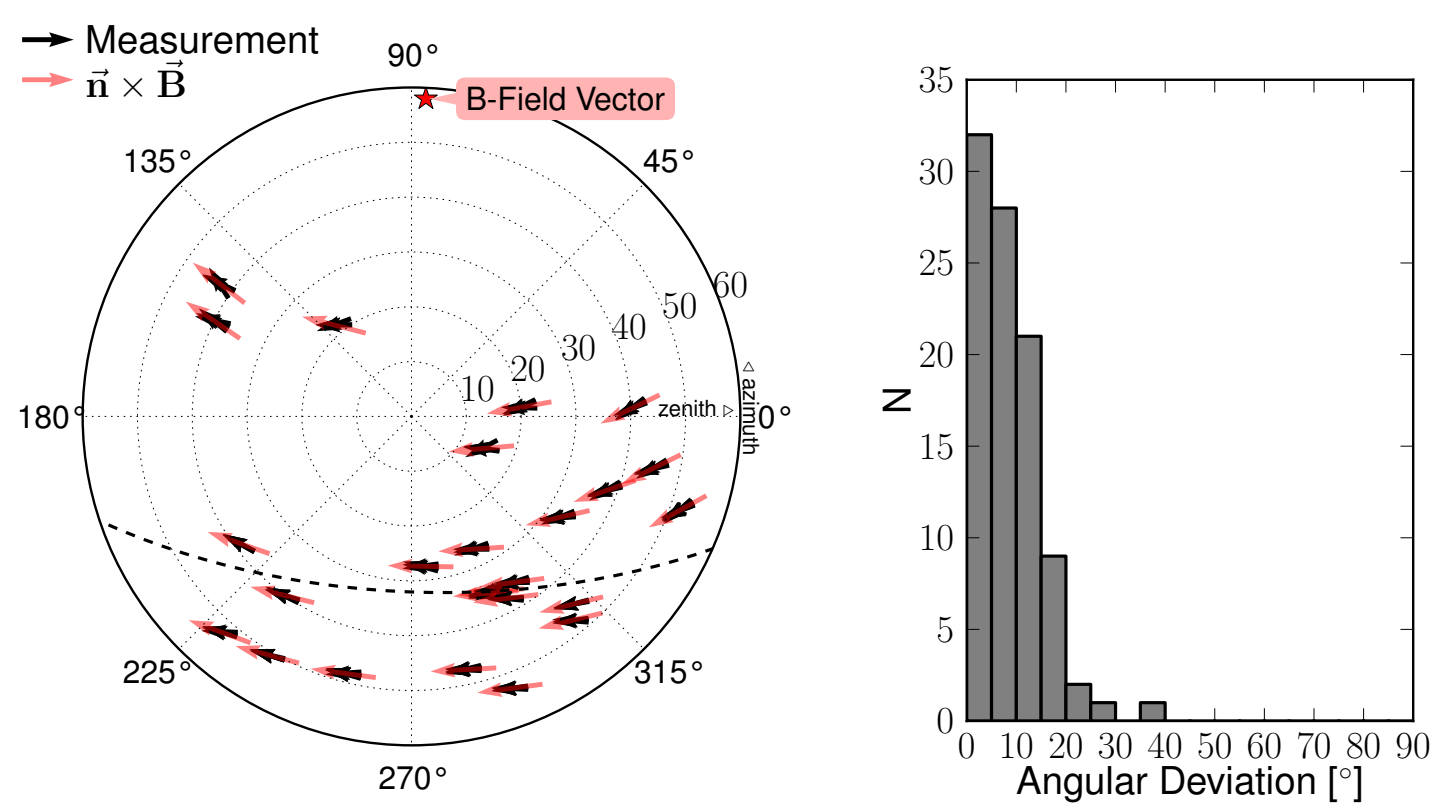

Figure 5: Polar skyplot of measured polarizations (horizontal components) in comparison to the $\vec{n} \times \vec{B}$ calculation (left). All vectors are normalized to unity. Note that at least three black vectors are plotted on top of each other for a specific incoming direction according to the multiple measurements of the electric field vector at the different radio stations triggered in the event. The dashed line indicates incoming directions perpendicular to the magnetic field vector (red star) at the AERA site. On the right, the angular deviation between the $\vec{n} \times \vec{B}$ vectors and the measured tree-dimensional polarization vectors is histogrammed.

The contribution of a radio emission due to a charge excess in the air shower [10] which leads to a fundamentally different polarization signature has been analyzed based on AERA data. It was found that the charge excess emission contributes to the total signal amplitude in average with a relative strength of $a=14 \pm 2 \%$ with respect to the geomagnetic emission [11].

\section{Radio Energy Measurements}

The radio measurement of the primary cosmic rays energy is a challenging task as the observed total radio emission is a superposition of different emission mechanisms whose interplay and geometrical dependencies are not fully understood yet. Nevertheless, an energy estimator can be formulated based on the model prediction that the radio amplitude $\left|\vec{E}_{\text {Model }}\right|$ is composed of the two identified contributions, geomagnetic $\vec{e}_{\mathrm{GM}}$ and charge excess emission $\vec{e}_{\mathrm{CE}}$

$$
\left|\vec{E}_{\text {Model }}\right| \propto\left|\sin (\alpha) \vec{e}_{\mathrm{GM}}+a \vec{e}_{\mathrm{CE}}\right| .
$$

A corrected radio signal amplitude $S_{\text {Corr. }}$, which takes the scaling of the geomagnetic emission with the incoming direction of the shower (cf. eq. 5.1) and the observed relative strength of the charge excess contribution $a$ into account, is obtained by scaling the measured radio amplitude $S$ with the model prediction, $S_{\text {Corr }}=S /\left|\vec{E}_{\text {Model }}\right|$.

The radio amplitude shows a characteristic falloff with increasing lateral distance to the shower axis. This is considered by fitting e.g. an exponential lateral distribution function to the corrected 
radio amplitudes observed at different distances to the shower axis by the individual stations. The final radio energy estimator is then defined as the radio amplitude evaluated at a fixed distance from the shower axis in the measured lateral distribution function.

The radio energy estimator can then be calibrated with the energy measurement of the Auger baseline detectors. A publication which follows the described method and includes a calibration with the Auger surface detector is currently under preparation.

\section{Conclusions}

With the digital radio array AERA, a pathfinder to explore the potential of radio detection of cosmic ray induced air showers has been commissioned and has been running stably since 2011 . Hardware and signal processing strategies have been developed to enable self-triggered radio detection of cosmic rays in a background dominated environment. New methods to calibrate the radio detector stations including the radio antennas have been applied to guarantee a reliable reconstruction of the radio emission from air showers.

High-precision polarization measurements have already delivered important contributions to the understanding of radio emission mechanisms including second order effects. The reconstruction of the cosmic ray energy provides an important step towards measuring all important cosmic ray properties with the radio technique. Beyond this, several methods to detect the mass of the cosmic ray with AERA are currently under investigation.

\section{Acknowledgements}

The successful installation, commissioning, and operation of the Pierre Auger Observatory would not have been possible without the strong commitment and effort from the technical and administrative staff in Malargüe.

\section{References}

[1] H. J. Mathes for the Pierre Auger Collaboration, 32nd ICRC, Beijing, China, 2011, arXiv:1107.4807

[2] F. Sanchez for the Pierre Auger Collaboration, 32nd ICRC, Beijing, China, 2011, arXiv:1107.4807

[3] K. Weidenhaupt for the Pierre Auger Collaboration, Acta Polytechnica, Vol. 53 Supplement, 2013

[4] J. L. Kelley for the Pierre Auger Collaboration, Proc. 32th ICRC, Beijing, China, 2011, arXiv: 1107.4807

[5] C. Rühle, PhD Thesis, Karlsruhe, 2013

[6] NEC2++, http://elec.otago.ac.nz/w/index.php/Necpp

[7] P. Abreu et al, Nucl.Instrum.Meth. A635, 92, 2011

[8] F. Schröder et al., Proc. 5th ARENA, Erlangen, Germany, 2012, AIP Conf. Proc. 1535, pp. 89-93

[9] D. Ardouin et al.,Geomagnetic origin of the radio emission from cosmic ray induced air showers observed by CODALEMA, arXiv:0901.4502

[10] G. Askaryan, Soviet Phys. JETP Lett. (USSR) 14, 441, 1962

[11] A. Aab et al., Phys. Rev. D 89, 2014 